\title{
Tratamentos osmóticos e térmicos para controle de fungos em sementes de grumixameira (Eugenia brasiliensis Lam.) e pitangueira (Eugenia uniflora L.)
}

\author{
Cibelle Ferreira Françoso ${ }^{1}$ e Claudio José Barbedo ${ }^{2,3}$
}

Recebido: 28.05.2013; aceito: 20.05.2014

\begin{abstract}
Osmotic and heat treatments to control fungi associated with seeds of Brazilian cherry (Eugenia brasiliensis Lam.) and Surinam cherry (Eugenia uniflora L.)). Seeds of Brazilian cherry (Eugenia brasiliensis Lam.) and Surinam cherry (Eugenia uniflora L.) are intolerant to desiccation and their high water content allows the proliferation of fungi, accelerating their deterioration. Due to the lack of fungicides registered for such species further studies on alternative methods should be carried out. The present work was aimed at analyzing the effects of heat and osmotic treatments on the mycota and seed viability of Brazilian cherry and Surinam cherry. We analyzed the effect of heat treatment (55 and $60^{\circ} \mathrm{C}$ for 30,120 , or 150 minutes) and osmotic treatment (polyethylene glycol solutions at -3.4 and $-4.0 \mathrm{MPa}$ ), each tested individually or in combination and also with or without reapplication throughout seed storage. Our results showed that heat treatment was more efficient than osmotic treatment for most fungi, except for Fusarium sp. The combination of both treatments produced favorable results and their reapplication may enhance the control of seed-borne fungi during storage.
\end{abstract}

Keywords: alternative control, conservation, seed pathology

RESUMO - (Tratamentos osmóticos e térmicos para controle de fungos em sementes de grumixameira (Eugenia brasiliensis Lam.) e pitangueira (Eugenia uniflora L.)). As sementes de grumixama (Eugenia brasiliensis Lam.) e pitanga (Eugenia uniflora L.) são intolerantes à dessecação e seu elevado teor de água favorece a proliferação de fungos que podem acelerar sua deterioração. A falta de fungicidas registrados para tais espécies requer estudos sobre métodos alternativos. O objetivo deste trabalho foi determinar o efeito de tratamentos térmicos e osmóticos na incidência de fungos e na viabilidade de sementes de grumixama e pitanga. Foram analisados os efeitos de tratamentos térmicos $\left(55\right.$ e $60{ }^{\circ} \mathrm{C}$ por 30,120 ou 150 minutos $)$ e osmóticos (soluções de polietileno glicol a -3,4 e -4,0 MPa) isolados ou associados e, ainda, com ou sem reaplicação durante o armazenamento das sementes. Verificou-se que o tratamento térmico foi mais eficiente que o osmótico na redução da maioria dos fungos encontrados, exceto Fusarium sp. A associação dos dois tipos de tratamento produz resultados favoráveis e a reaplicação dos mesmos pode ampliar o controle dos fungos ao longo do armazenamento.

Palavras-chave: conservação, controle alternativo, patologia de sementes

\section{Introdução}

Espécies frutíferas nativas do Brasil vêm despertando interesse econômico cada vez maior face à crescente diversidade de usos descobertos para cada parte da planta. Além de potencialidades de uso alimentício e farmacêutico, espécies do gênero Eugenia (Myrtaceae) foram recentemente incluídas na cadeia de produção de cosméticos (Schmeda-Hirschmann et al. 1987, Delgado et al. 2010, Amador \& Barbedo 2011, Delgado \& Barbedo 2012). Contudo, o cultivo em larga escala dessas espécies depende do melhor conhecimento de diversos fatores, dentre os quais a conservação da viabilidade de suas sementes em armazenamento figura entre os principais. Sementes de pitangueira (Eugenia uniflora L.) e de grumixameira (E. brasiliensis Lam.) não toleram desidratação (Delgado \& Barbedo 2007), sendo, portanto, classificadas como recalcitrantes. Sementes recalcitrantes favorecem a proliferação de microrganismos, principalmente os fungos, que aceleram consideravelmente a velocidade de deterioração (Berjak 1996), como foi observado

1. Programa de Pós-Graduação em Biodiversidade Vegetal e Meio Ambiente do Instituto de Botânica

2. Instituto de Botânica, Núcleo de Pesquisa em Sementes, Avenida Miguel Stéfano, no 3.687, 04301-902 São Paulo, SP, Brasil

3. Autor para correspondência: claudio.barbedo@pq.cnpq.br 
para sementes de Inga vera (Parisi et al. 2013). Essa atuação dos fungos na deterioração durante o armazenamento de sementes tem sido foco de alguns poucos trabalhos nos últimos anos (Sutherland et al. 2002, Oliveira et al. 2011) e tem-se verificado que há diferenças em relação ao conhecimento clássico descrito para sementes tolerantes. A proliferação de fungos em sementes e a deterioração destas pode ser uma via de mão dupla, ou seja, tanto a deterioração da semente facilita o crescimento dos fungos quanto este crescimento pode acelerar o processo de deterioração (Berjak 1996). Portanto, a remoção da principal fonte de inóculo dos fungos poderia propiciar o aumento da longevidade das sementes intolerantes à dessecação.

$\mathrm{O}$ controle químico tem sido a forma mais comumente utilizada para o tratamento de sementes (Mendes et al. 2001), mas requer a manutenção de registro de produtos para a espécie, o que não ocorre para as espécies florestais (Parisi et al. 2011). Os tratamentos físicos, como a termoterapia (em água quente) e o condicionamento osmótico (em soluções de potencial hídrico controlado), por sua vez, podem controlar os patógenos, mas dependem do conhecimento da sensibilidade diferencial a tais estresses (térmico ou hídrico) entre a semente e o patógeno.

O tratamento térmico já é amplamente conhecido e utilizado para diversas espécies e estruturas vegetais, como em sementes de tomateiro, para controle de Xanthomonas campestris pv. vesicatoria (Silva et al. 2002) e Vírus do Mosaico do Tomate (Silva et al. 2011), mas o condicionamento osmótico, como método para controle de fungos, tem sido analisado apenas mais recentemente (Oliveira et al. 2011). A técnica do armazenamento das sementes em soluções osmóticas foi utilizada para sementes de Inga vera, mas com o objetivo de reduzir o metabolismo intenso e desordenado dos embriões por meio da regulação da mobilização da água (Andréo et al. 2006). Contudo, esse método pode auxiliar no controle de patógenos, uma vez que o controle da atividade da água pode inibir ou mesmo cessar o crescimento dos fungos (Oliveira et al. 2011). A associação dos dois tratamentos, térmico e osmótico, poderia ampliar ainda mais a diversidade e eficiência de tratamentos alternativos aos químicos em sementes. Porém, a falta de proteção residual contra a recontaminação por patógenos oportunistas e injúrias promovidas no hospedeiro representa, atualmente, a maior limitação ao uso da termoterapia e do tratamento osmótico (Oliveira et al. 2011). Assim, a reaplicação periódica de tratamentos poderia ampliar o efeito sobre o controle dos fungos associados às sementes.

No presente trabalho objetivou-se avaliar o efeito de tratamentos térmicos e osmóticos, quando aplicados isolados e associados, bem como os efeitos da reaplicação desses tratamentos, no controle dos fungos e na manutenção da viabilidade das sementes, visando a contribuir para o armazenamento de sementes e a produção de mudas de grumixameira e pitangueira.

\section{Material e métodos}

Os frutos de grumixameira (Eugenia brasiliensis Lam.) e pitangueira (Eugenia uniflora L.) foram obtidos nos meses de dezembro de 2011 e janeiro de 2012, respectivamente, de matrizes plantadas no Jardim Botânico de São Paulo, São Paulo, SP (233' S e $\left.46^{\circ} 37^{\prime} \mathrm{W}\right)$. O processo de extração e beneficiamento das sementes consistiu da extração manual da polpa, eliminação de sementes danificadas por insetos e imaturas, e armazenamento em sacos de polietileno, em câmara fria a $7{ }^{\circ} \mathrm{C}$ (Kohama et al. 2006), até a instalação dos experimentos, não ultrapassando 15 dias.

Inicialmente (após a extração e o beneficiamento) e após os tratamentos e os períodos de armazenamento (descritos abaixo), as sementes foram analisadas quanto à sua qualidade fisiológica (germinação e sementes germináveis) e sanitária (teste de sanidade).

O teste de germinação foi conduzido em rolos de papel Germitest, previamente umedecido (Brasil 2009), mantidos em câmaras de germinação, reguladas para $25 \pm 1{ }^{\circ} \mathrm{C}$, com luz contínua e $100 \%$ de umidade relativa do ar (Delgado \& Barbedo 2007), tendo sido utilizadas quatro repetições de 12 sementes. As avaliações foram realizadas a cada três dias durante 70 dias, registrando-se a porcentagem de sementes com emissão de raiz primária (viabilidade), doravante denominadas sementes germináveis, e a porcentagem de plântulas normais desenvolvidas, doravante denominada germinação (Kohama et al. 2006).

O teste de sanidade foi realizado pelo método de incubação em papel de filtro (Brasil 2009). As sementes foram distribuídas equidistantemente em placas de Petri $(90 \times 15 \mathrm{~mm})$, contendo três folhas de papel de filtro umedecidas com água destilada e foram, então, incubadas por sete dias a $20 \pm 2{ }^{\circ} \mathrm{C}$ e fotoperíodo de 12 horas. Utilizaram-se quatro repetições de 12 sementes, com seis sementes por placa (duas placas por repetição). A identificação e a contagem dos fungos foram realizadas examinando-se as colônias 
fúngicas desenvolvidas nas sementes com auxílio de microscópio estereoscópico. Para o cálculo da incidência de fungos nas sementes, em porcentagem, cada fungo foi analisado individualmente. Em cada análise foi considerado o número de sementes com presença do fungo analisado em relação ao total de sementes incubadas. Em alguns casos, a identificação do fungo foi complementada pela visualização das características morfológicas em microscópio óptico (Barnett \& Hunter 1999). A incidência de Penicillium sp., Fusarium sp., Pestalotiopsis sp., Botrytis sp. e Cladosporium sp., que se mostraram recorrentes e persistentes no armazenamento de espécies de Eugenia sp. (Oliveira et al. 2011), foi utilizada como principal referencial para a análise da eficiência dos tratamentos no controle da micota.

Foram utilizados dois tratamentos térmicos (Term-1 e Term-2) e dois tratamentos osmóticos (Osm-1 e Osm-2), além da ausência de tratamento térmico (Term-0) e osmótico (Osm-0). A Testemunha foi caracterizada pelas sementes que não receberam quaisquer dos tratamentos, térmico ou osmótico (Term-0 e Osm-0). Em função da diferença na sensibilidade térmica das sementes das duas espécies (Oliveira et al. 2011), foram empregados tratamentos térmicos distintos para as espécies. Dessa forma, Term-1 correspondeu ao tratamento de $55^{\circ} \mathrm{C}$ por 30 minutos, para E. brasiliensis e por 120 minutos, para E. uniflora; Term-2 correspondeu a $55{ }^{\circ} \mathrm{C}$ por 150 minutos, para $E$. brasiliensis e $60^{\circ} \mathrm{C}$ por 120 minutos, para E. uniflora.

Os tratamentos térmicos foram realizados imergindo-se as sementes em água destilada, em béqueres de vidro acondicionados em estufa com circulação de ar, nas temperaturas e períodos descritos anteriormente. Foi utilizada a proporção de 1:5 entre o peso das sementes e o peso da água do tratamento. Os béqueres foram periodicamente agitados e, ao término do período de exposição, as sementes foram resfriadas com água destilada a temperatura ambiente por duas horas e depositadas sobre papel de filtro para a retirada do excesso de água superficial.

Os tratamentos osmóticos corresponderam a potenciais de -3,4 MPa (Osm-1) e -4,0 MPa (Osm-2) para ambas as espécies. Para tanto, incubaram-se as sementes em solução de polietileno glicol 6000 (PEG) por sete dias, em caixas tipo gerbox, a $7{ }^{\circ} \mathrm{C}$. Após os tratamentos osmóticos, as sementes foram lavadas por cinco vezes consecutivas em água destilada, para remoção da solução residual de PEG. As soluções osmóticas foram preparadas ajustando-se a concentração do sal em função da temperatura de incubação (Michel \& Kauffmann 1973), com aferição em analisador de potencial hídrico WP4 (Decagon, Pullmann, USA).

Analisou-se, também, a associação de ambos os tipos de tratamento. A associação de tratamentos foi feita realizando-se inicialmente o tratamento térmico; após o resfriamento, descrito anteriormente, as sementes foram incubadas no tratamento osmótico, seguindo-se o mesmo procedimento dos tratamentos isolados.

Após a aplicação dos tratamentos, as sementes foram armazenadas em sacos de polietileno, em câmara fria a $7{ }^{\circ} \mathrm{C}$ (Kohama et al. 2006) por 30 dias. Após esse período, as amostras de sementes de cada tratamento foram divididas em duas amostras e uma delas foi submetida à reaplicação do tratamento que havia recebido inicialmente. Ambas foram, então, armazenadas por mais 30 dias.

O tratamento químico foi utilizado apenas como referencial de controle; para tanto, as sementes foram tratadas com o fungicida carbendazin + thiram, na dosagem e metodologia recomendada pelo fabricante para espécies agrícolas $\left(300 \mathrm{~mL} 100 \mathrm{~kg}\right.$ de sementes $\left.{ }^{-1}\right)$, baseando-se em resultados anteriores desenvolvidos com sementes de Eugenia (Oliveira et al. 2011). Sementes e produto foram colocados em saco plástico, que foi agitado até distribuição uniforme do produto na superfície das sementes.

O delineamento experimental para todos os experimentos foi o inteiramente casualizado, com quatro repetições. Para as avaliações de qualidade fisiológica adotou-se esquema fatorial $3 \times 3 \times 2$ (tratamentos térmicos $\times$ tratamentos osmóticos $\times$ reaplicação), dentro de cada período de armazenamento. Os dados obtidos foram submetidos à análise de variância (teste $F, p<0,05$ ), sem transformação de dados, isoladamente para cada espécie e cada período de armazenamento. As médias das variáveis fisiológicas foram comparadas entre si pelo teste de Tukey (Santana \& Ranal 2004) e as médias das variáveis de sanidade foram apresentadas com seus respectivos desvios-padrão.

\section{Resultados}

Os fungos mais frequentemente encontrados nas sementes de grumixameira e pitangueira foram os dos gêneros Penicillium e Fusarium (figuras 1 e 2), mas também foram encontrados Pestalotiopsis, Cladosporium e Botrytis (figuras 3 e 4). O tratamento químico (fungicida) apresentou baixa eficiência inicial 
de redução dos fungos, demonstrando a dificuldade no controle de fungos associados a sementes de Eugenia.

$\mathrm{O}$ tratamento térmico mostrou-se eficiente em reduzir a incidência de Cladosporium sp. em ambas as espécies de Eugenia e, também, a incidência de Penicillium sp. em E. brasiliensis e a incidência de Fusarium sp., Pestalotiopsis sp. e Botrytis sp. em E. uniflora. Contudo, o tratamento térmico foi ineficiente ou até prejudicial em algumas combinações como, por exemplo, Term-2 para controle de

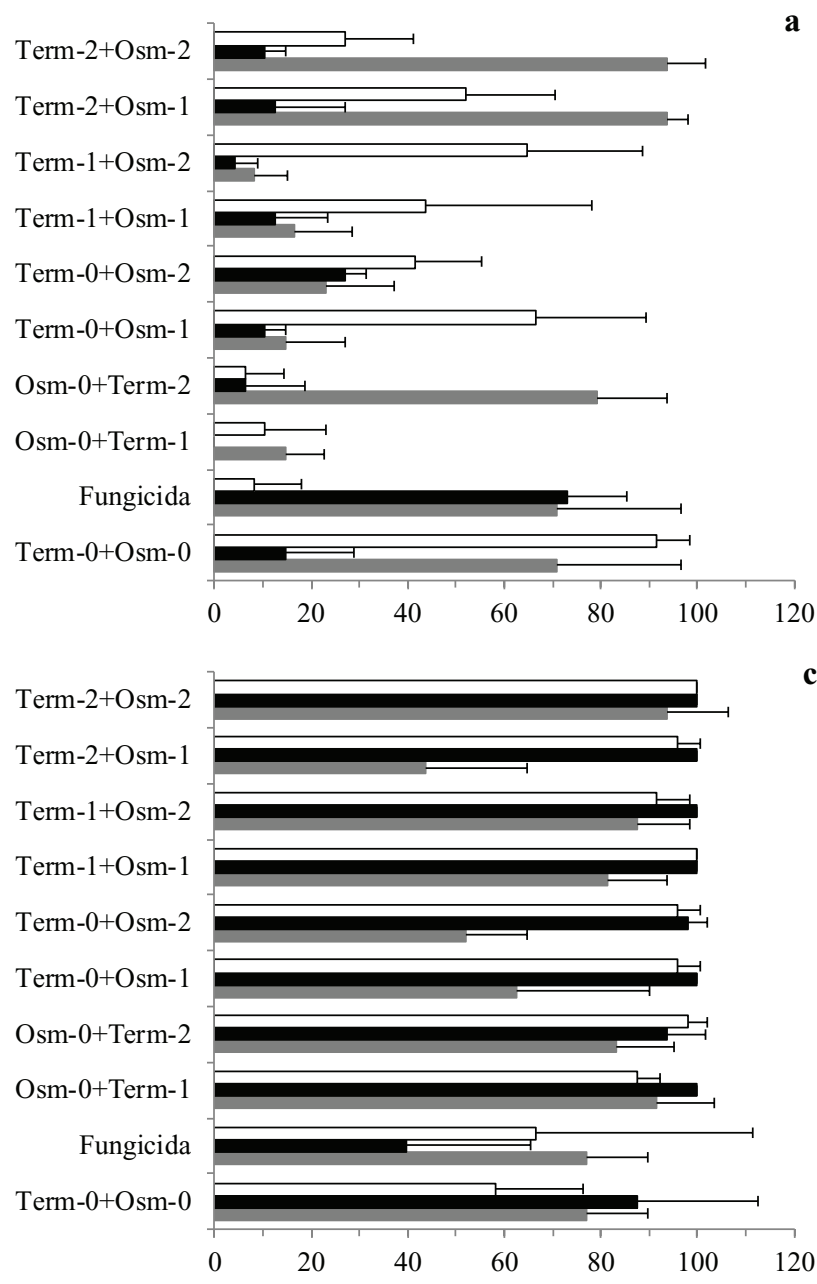

Penicillium sp. em E. brasiliensis (figura 1a) e Term-1 e Term-2 para controle de Penicillium sp. em E. uniflora (figura 2a, c).

O tratamento osmótico, por sua vez, não reduziu a incidência de Fusarium sp. em ambas as espécies de Eugenia (figuras 1 e 2), tampouco de Pestalotiopsis sp. em E. uniflora (figura 4a), mas mostrou-se eficiente para o controle de Penicillium sp. (figuras 1 e 2), Cladosporium sp. e Botrytis sp. (figuras 3 e 4) nas duas espécies de Eugenia. a

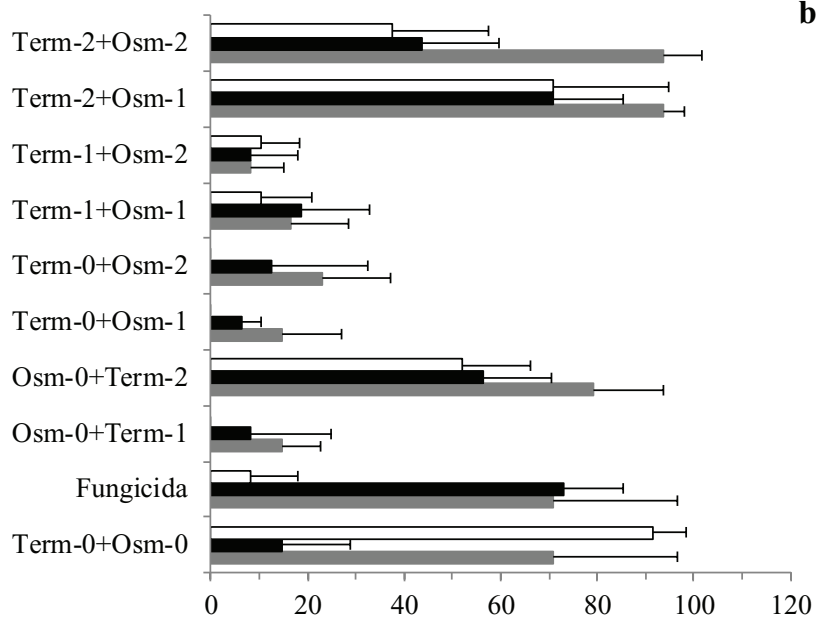

c

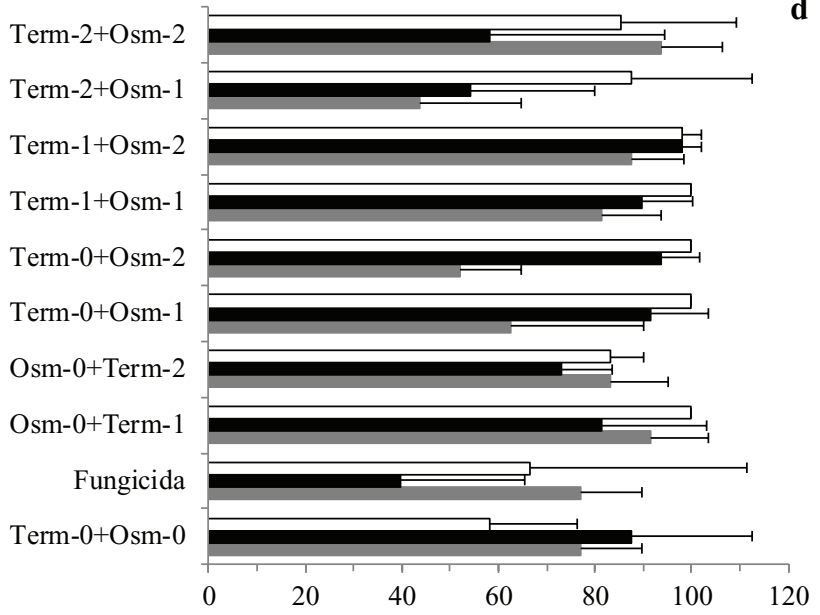

Incidência de Fungos (\%)

Figura 1. Incidência (\%) de Penicillium sp. (a e b) e Fusarium sp. (c e d) em sementes de Eugenia brasiliensis submetidas aos tratamentos térmicos (Term-0: sem tratamento térmico; Term-1: $55^{\circ} \mathrm{C} / 30 \mathrm{~min}$; Term-2: $55^{\circ} \mathrm{C} / 150 \mathrm{~min}$ ) e osmóticos (Osm- 0 : sem tratamento osmótico; Osm-1: -3,4 MPa/7dias; Osm-2: -4,0 MPa/7dias), com (b e d) ou sem (a e c) reaplicação após 30 dias de armazenamento, bem como as sementes tratadas com fungicida carbendazin + tiram. Barras cinzas: sementes não armazenadas; barras pretas: após 30 dias de armazenamento; barras brancas: após 60 dias. São apresentadas as médias e os desvios-padrão.

Figure 1. Incidence (\%) of Penicillium sp. (a and b) and Fusarium sp. (c and d) on seeds of E. brasiliensis after heat treatments (Term-0: without heat treatment; Term-1: $55^{\circ} \mathrm{C} / 30 \mathrm{~min}$; Term-2: $55^{\circ} \mathrm{C} / 150 \mathrm{~min}$ ) and osmotic treatment (Osm-0: without osmotic treatment; Osm-1: $-3.4 \mathrm{MPa} / 7$ days; Osm-2: $-4.0 \mathrm{MPa} / 7$ days), with (b and d) or without (a and c) reapplication after 30 days, as well as the seeds treated with the fungicide carbendazim + thiram. Gray bars: non-stored seeds; black bars: after 30 days of storage; white bars: after 60 days of storage. Values are means with standard deviations. 
A associação dos tratamentos, térmico e osmótico, em geral, não demonstrou vantagem em relação aos tratamentos isolados. Contudo, deve-se ressaltar que a associação de Term-2 com Osm-1 ou com Osm-2 melhorou o controle de Fusarium sp. em sementes de E. uniflora, em relação aos tratamentos térmicos isolados, que foram os únicos a controlar esse fungo (figura 2c).

O armazenamento das sementes pouco modificou a incidência de Fusarium sp., que continuou em elevada frequência (figuras 1 e 2); a incidência de Penicillium sp. permaneceu baixa em E. uniflora e, em E. brasiliensis, reduziu aos 30 dias mas voltou a se elevar aos 60 dias (figura 1a). A incidência de Pestalotiopsis sp. permaneceu constante até os 60 dias em E. uniflora (figura 4a) e, em E. brasiliensis, aumentou até os 30 dias de armazenamento, desaparecendo aos 60 dias (figura 3a). A incidência dos demais fungos foi, em geral, reduzida ao longo do armazenamento das sementes de ambas as espécies de Eugenia.

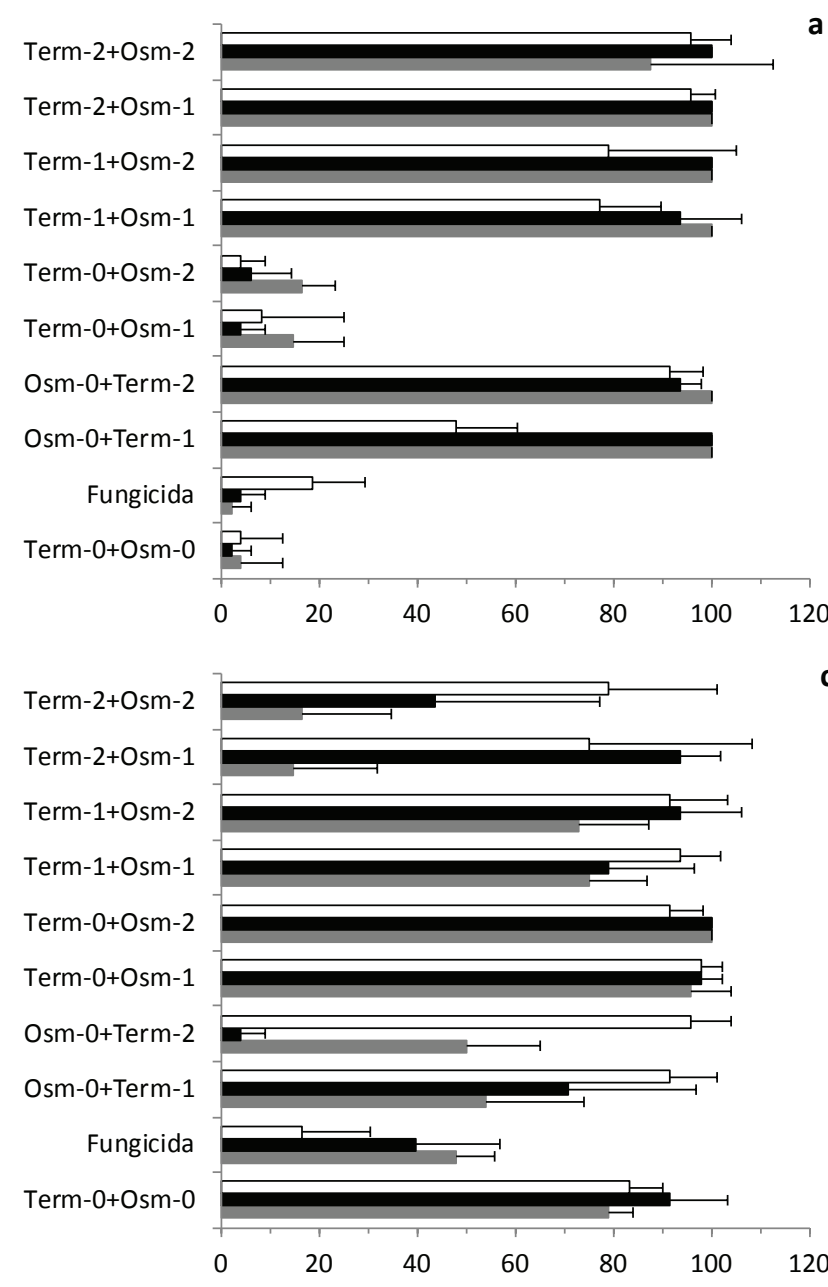

a

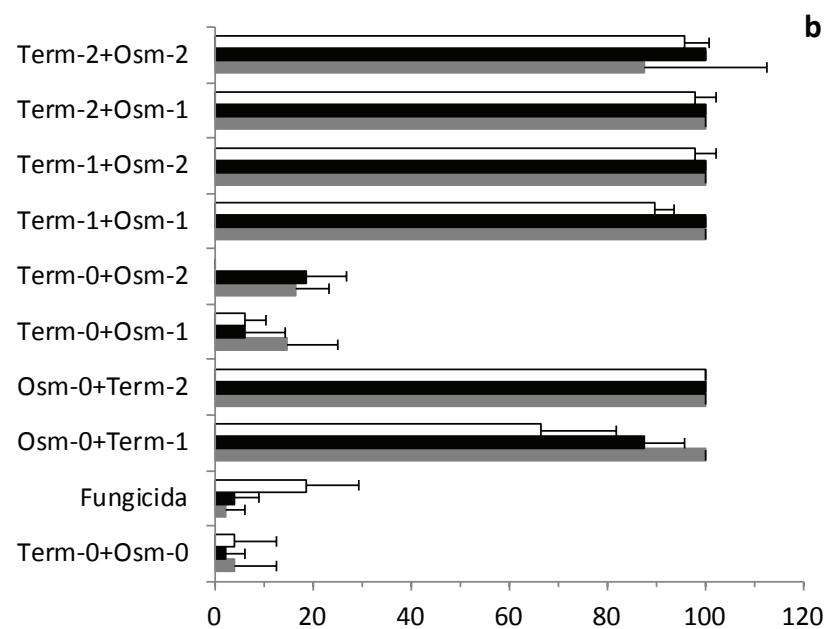

C

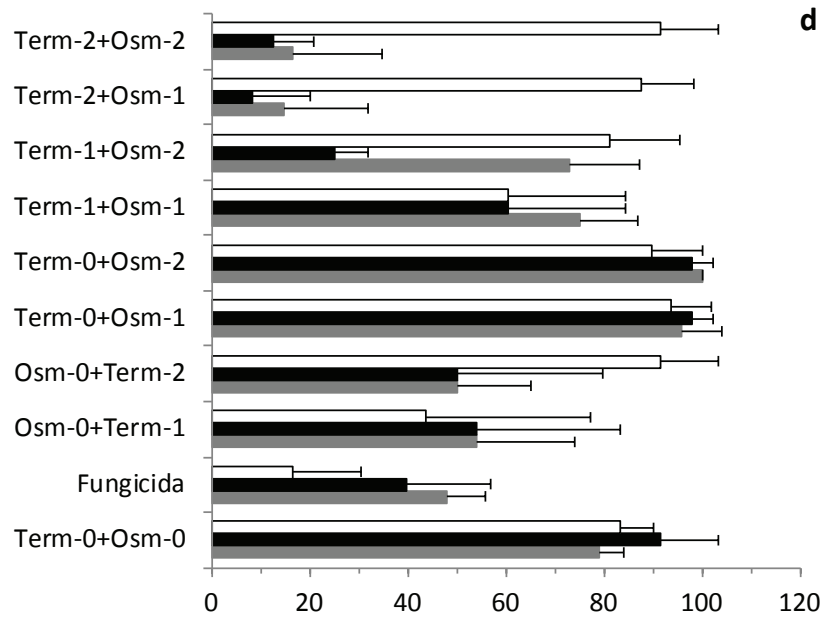

Incidência de Fungos (\%)

Figura 2. Incidência de Penicillium sp. (a e b) e Fusarium sp. (c e d) em sementes de Eugenia uniflora submetidas aos tratamentos térmicos (Term-0: sem tratamento térmico; Term-1: $55{ }^{\circ} \mathrm{C} / 120 \mathrm{~min}$; Term-2: $60{ }^{\circ} \mathrm{C} / 120 \mathrm{~min}$ ) e osmóticos (Osm-0: sem tratamento osmótico; Osm-1: -3,4 MPa/7dias; Osm-2: -4,0 MPa/7dias), com (b e d) ou sem (a e c) reaplicação após 30 dias de armazenamento, bem como as sementes tratadas com fungicida carbendazin + tiram. Barras cinzas: sementes não armazenadas; barras pretas: após 30 dias de armazenamento; barras brancas: após 60 dias. São apresentadas as médias e os desvios-padrão.

Figure 2. Incidence (\%) of Penicillium sp. (a and b) and Fusarium sp. (c and d) on seeds of Eugenia uniflora after heat treatments (Term-0: without heat treatment; Term- $1: 55^{\circ} \mathrm{C} / 120 \mathrm{~min}$; Term-2: $60^{\circ} \mathrm{C} / 120 \mathrm{~min}$ ) and osmotic treatment (Osm- 0 : without osmotic treatment; Osm-1: $-3.4 \mathrm{MPa} / 7$ days; Osm-2: $-4.0 \mathrm{MPa} / 7$ days), with (b and d) or without (a and c) reapplication after 30 days, as well as the seeds treated with the fungicide carbendazim + thiram. Gray bars: non-stored seeds; black bars: after 30 days of storage; white bars: after 60 days of storage. Values are means with standard deviations. 

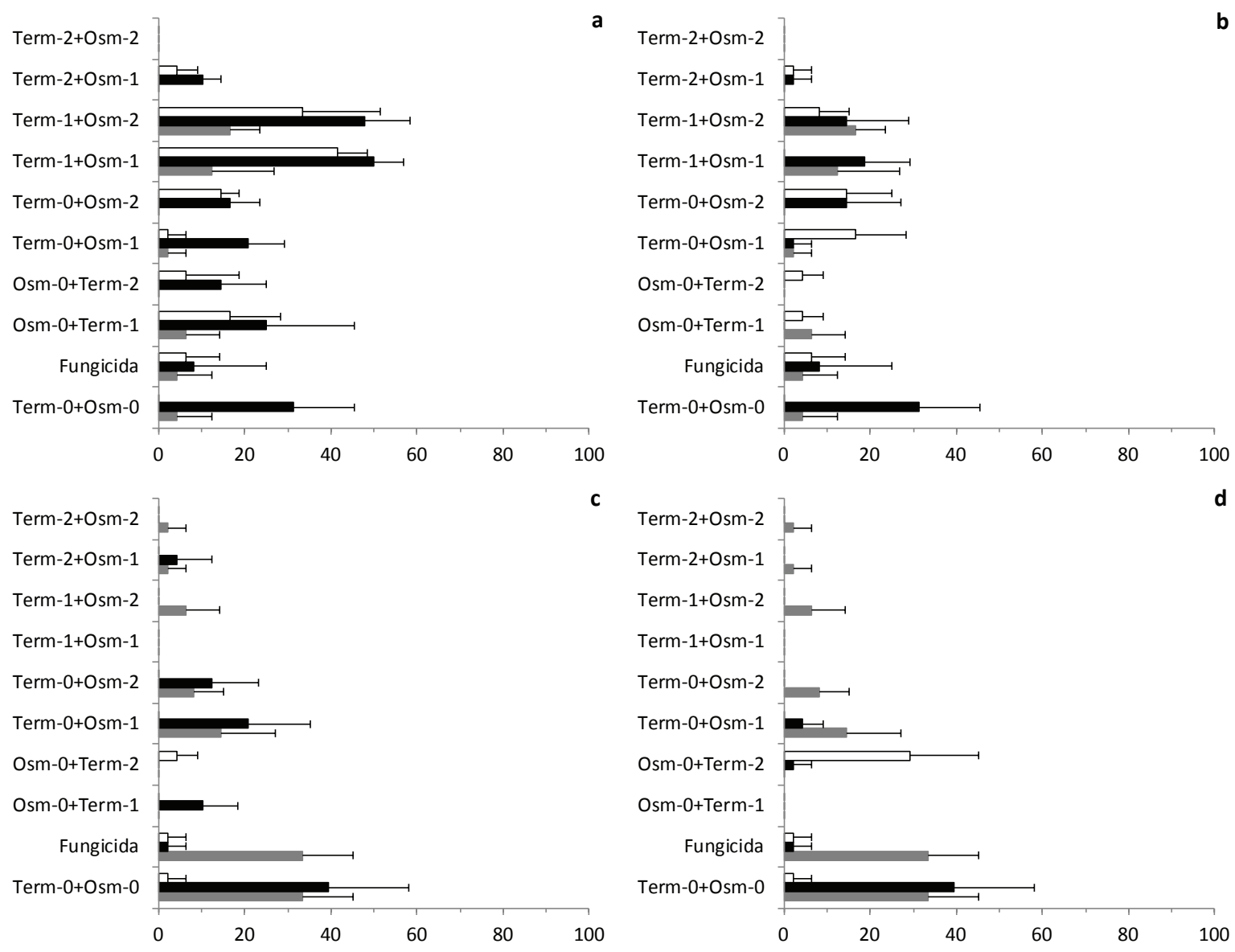

Term-1+Osm-2
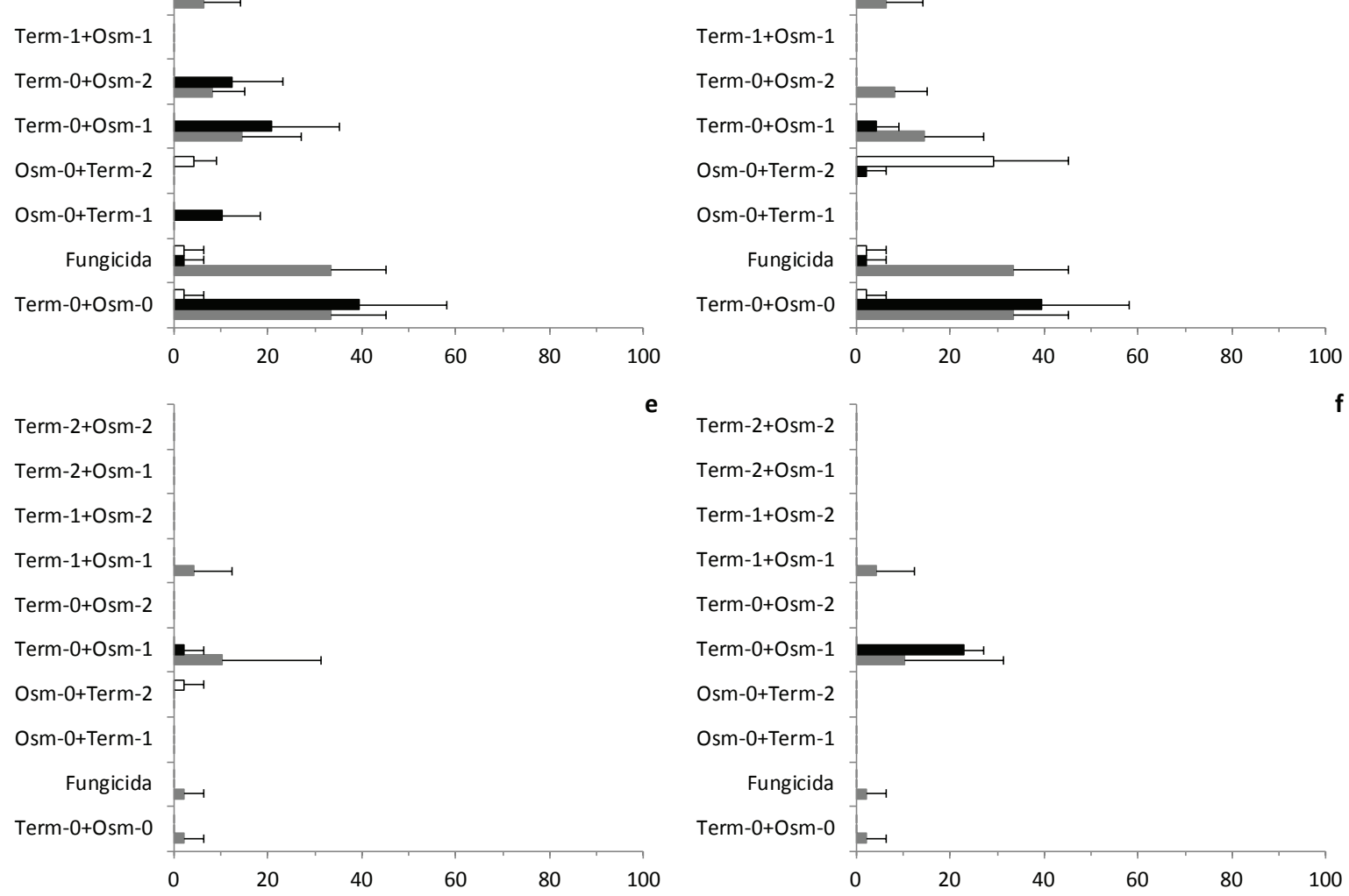

Incidência de Fungos (\%)

Figura 3. Incidência de Pestalotiopsis sp. (a e b), Cladosporium sp. (c e d) e Botrytis sp. (e e f) em sementes de Eugenia brasiliensis submetidas aos tratamentos térmicos (Term- 0 : sem tratamento térmico; Term- $1: 55^{\circ} \mathrm{C} / 30 \mathrm{~min}$; Term-2: $\left.55^{\circ} \mathrm{C} / 150 \mathrm{~min}\right)$ e osmóticos $(\mathrm{Osm}-0$ : sem tratamento osmótico; Osm-1: -3,4 MPa/7dias; Osm-2: -4,0 MPa/7 dias), com (b, d e f) ou sem (a, c e e) reaplicação após 30 dias de armazenamento, bem como as sementes tratadas com fungicida carbendazin + tiram. Barras cinzas: sementes não armazenadas; barras pretas: após 30 dias de armazenamento; barras brancas: após 60 dias. São apresentadas as médias e os desvios-padrão.

Figure 3. Incidence (\%) of Pestalotiopsis sp. (a and b), Cladosporium sp. (c and d) and Botrytis sp. (e and f) on seeds of Eugenia brasiliensis after heat treatments (Term-0: without heat treatment; Term-1: $55^{\circ} \mathrm{C} / 30 \mathrm{~min}$; Term-2: $\left.55{ }^{\circ} \mathrm{C} / 150 \mathrm{~min}\right)$ and osmotic treatment $(\mathrm{Osm}-0$ : without osmotic treatment; Osm-1: -3.4 MPa/7days; Osm-2: -4.0 MPa/7days), with (b, d, and f) or without (a, c, and e) reapplication after 30 days, as well as the seeds treated with the fungicide carbendazim + thiram. Gray bars: non-stored seeds; black bars: after 30 days of storage; white bars: after 60 days of storage. Values are means with standard deviations. 

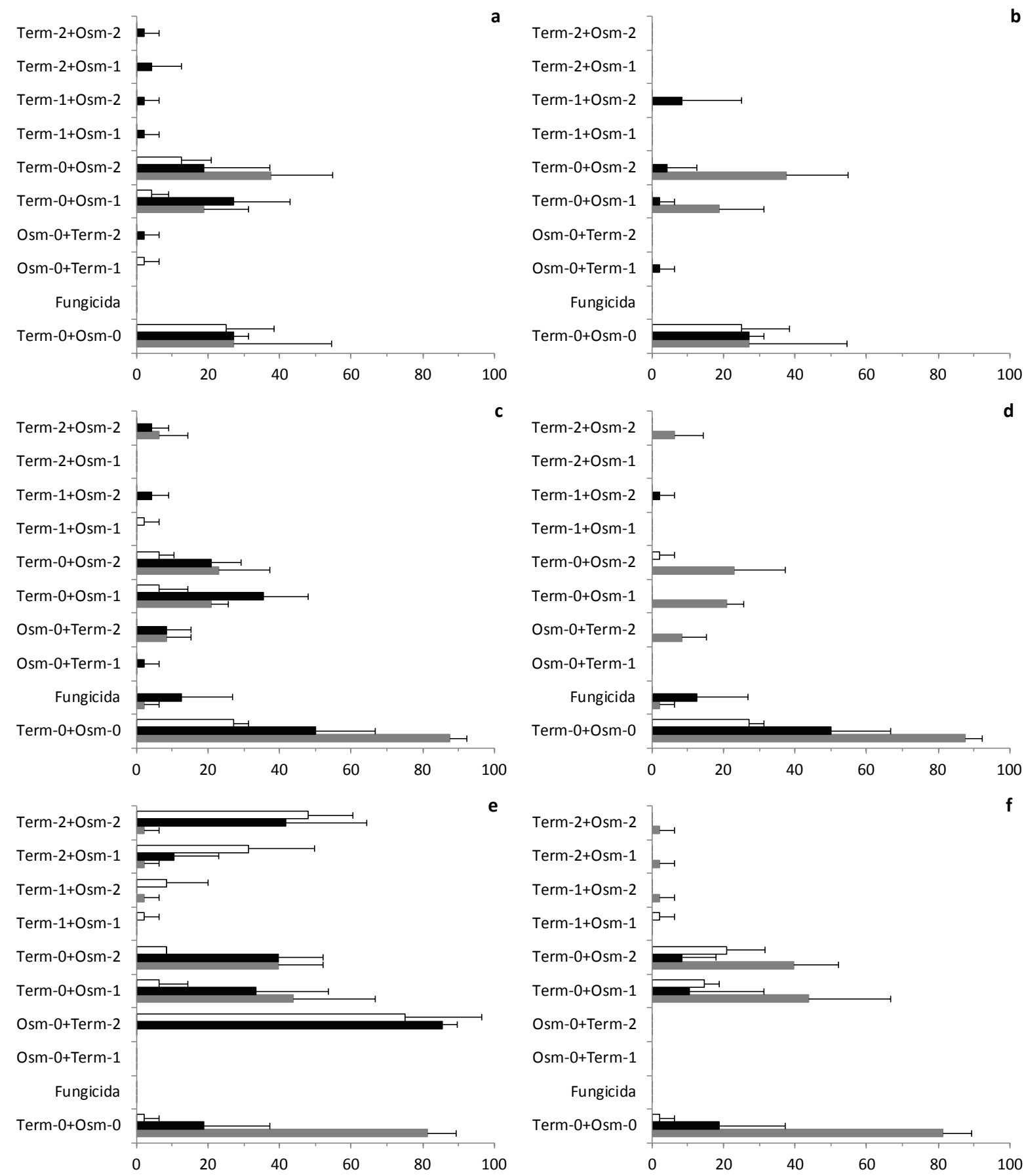

Incidência de Fungos (\%)

Figura 4. Incidência de Pestalotiopsis sp. (a e b), Cladosporium sp. (c e d) e Botrytis sp. (e e f) em sementes de Eugenia uniflora submetidas aos tratamentos térmicos (Term-0: sem tratamento térmico; Term-1: $55{ }^{\circ} \mathrm{C} / 120 \mathrm{~min}$; Term-2: $60{ }^{\circ} \mathrm{C} / 120 \mathrm{~min}$ ) e osmóticos (Osm-0: sem tratamento osmótico; Osm-1: -3,4 MPa/7dias; Osm-2: -4,0 MPa/7dias), com (b,d e f) ou sem (a, c e e) reaplicação após 30 dias de armazenamento, bem como as sementes tratadas com fungicida carbendazin + tiram. Barras cinzas: sementes não armazenadas; barras pretas: após 30 dias de armazenamento; barras brancas: após 60 dias. São apresentadas as médias e os desvios-padrão.

Figure 4. Incidence (\%) of Pestalotiopsis sp. (a and b), Cladosporium sp. (c and d) and Botrytis sp. (e and f) on seeds of Eugenia uniflora after heat treatments (Term-0: without heat treatment; Term-1: $55^{\circ} \mathrm{C} / 120 \mathrm{~min}$; Term-2: $\left.60{ }^{\circ} \mathrm{C} / 120 \mathrm{~min}\right)$ and osmotic treatment (Osm-0: without osmotic treatment; Osm-1:-3.4 MPa/7days; Osm-2: -4.0 MPa/7days), with (b, d, and f) or without (a, c, and e) reapplication after 30 days, as well as the seeds treated with the fungicide carbendazim + thiram. Gray bars: non-stored seeds; black bars: after 30 days of storage; white bars: after 60 days of storage. Values are means with standad deviations. 
A incidência de Fusarium continuou sendo a de mais difícil controle ao longo do armazenamento pois, mesmo nos tratamentos em que se obteve algum controle inicial, o fungo voltou a apresentar elevadas incidências após 30 ou 60 dias. O fungicida foi o único tratamento que reduziu a incidência de Fusarium sp. ao longo do armazenamento e, mesmo assim, apenas para E. uniflora. Contudo, a reaplicação de Term-1 após 30 dias de armazenamento promoveu controle semelhante ao do fungicida (figura 2d). Para Penicillium, os tratamentos térmicos foram eficientes em manter a incidência baixa em $E$. brasiliensis, mas o mesmo não para as sementes de E. uniflora (figura 2a), para as quais o tratamento osmótico foi mais eficiente. Os tratamentos osmóticos foram eficientes em controlar a incidência de Penicillium sp. somente quando reaplicados após 30 dias de armazenamento (figura 1b).

A qualidade fisiológica das sementes de E. brasiliensis alterou-se muito pouco após quaisquer dos tratamentos térmicos e osmóticos, isolados ou associados, reaplicados ou não (tabela 1), sendo as maiores alterações oriundas dos tratamentos osmóticos isolados (Osm-1 e Osm-2). Por outro lado, as sementes de E. uniflora demonstraram grande sensibilidade aos tratamentos térmicos com a temperatura mais elevada (Term-2, $60{ }^{\circ} \mathrm{C}$, tabela 2), tanto isolados quanto em associação com os osmóticos.

\section{Discussão}

A ação de fungos acelerando a deterioração ou reduzindo o vigor de sementes intolerantes à dessecação durante $\mathrm{o}$ armazenamento foi demonstrada para algumas espécies (Mycock \& Berjak 1990, 1995, Berjak 1996, Calistru et al. 2000, Anguelova-Merhar et al. 2003). Verificou-se que o armazenamento obrigatoriamente úmido dessas sementes, diferentemente do que ocorre com sementes tolerantes à dessecação, favorece o desenvolvimento de fungos tradicionalmente classificados como fungos de campo. Nas sementes de Eugenia brasiliensis e E. uniflora do presente trabalho, tanto Penicillium sp. (fungo de armazenamento) quanto Fusarium sp. (de campo) foram observados durante todo o armazenamento.

Os fungicidas frequentemente têm se mostrado eficientes no controle de fungos associados às sementes durante o armazenamento, principalmente devido ao seu efeito residual (Calistru et al. 2000, Mendes et al. 2001, Bettiol \& Ghini 2003). Contudo, no presente trabalho esperava-se uma maior eficiência dos fungicidas no controle da maioria dos fungos associados às sementes. Tal fato já havia sido observado em sementes de Eugenia por Oliveira et al. (2011). Um dos possíveis fatores que pode ter contribuído para essa baixa eficiência é o fato das sementes serem recalcitrantes (ou ortodoxas imaturas, segundo Barbedo et al. 2013) e, portanto, necessitam ser mantidas com elevado teor de água. As sementes do presente trabalho mantiveram-se com teor de água entre 48 e 55\%. Segundo Dhingra (2005), a eficiência de um produto está relacionada a fatores como a dose aplicada do produto, sua formulação, a carga de inóculo do lote e, também, do teor de água das sementes que, quanto mais úmidas, maior a dificuldade na adesão do produto.

O tratamento térmico tem mostrado eficiência no controle de fungos em sementes de algumas espécies, provavelmente porque o calor destrói facilmente conídios e hifas, mas muitas espécies de fungos produzem esporos termo-resistentes (Splittstoesser \& Churey 1991). No presente trabalho, os tratamentos térmicos reduziram a incidência da maioria dos fungos presentes em sementes de E. brasiliensis e E. uniflora, exceto Penicillium sp. e Fusarium sp. Apesar da maioria dos microrganismos fitopatogênicos apresentar ponto térmico letal em temperaturas na faixa entre 45 a $60{ }^{\circ} \mathrm{C}$ (Civello et al. 1997), conídios de algumas espécies são muito mais resistentes, o que poderia explicar a presença daqueles dois fungos após os tratamentos térmicos.

De fato, particularmente para Fusarium sp. há relatos na literatura da formação de clamidósporos (estruturas de resistência) quando as condições ambientais são desfavoráveis. Esses clamidósporos podem ser formados em 2 a 3 dias após a germinação dos esporos e podem sobreviver por mais de 20 anos (Beckman \& Roberts 1995). Portanto, podem ter sido formados durante os tratamentos osmóticos, mas no caso dos térmicos, já deveriam estar presentes nas sementes. Tanaka et al. (2003), por exemplo, também observaram a resistência de Fusarium spp. ao tratamento em água a $55{ }^{\circ} \mathrm{C}$ por até 30 minutos e a $60{ }^{\circ} \mathrm{C}$ por 10 minutos. Por outro lado, Mendes et al. (2001) conseguiram reduzir significativamente a incidência de $F$. oxysporum em sementes ao aplicar o tratamento térmico de calor seco a $60{ }^{\circ} \mathrm{C}$ por $20 \mathrm{e}$ 30 minutos. Neste caso, é possível que não houvesse clamidósporos ou que estes possam ser controlados quando o calor aplicado é seco. É importante salientar, contudo, que as sementes das espécies estudadas são intolerantes à dessecação e, portanto, para manter sua viabilidade deve-se mantê-las úmidas. 
Tratamentos osmóticos por períodos prolongados, em sementes de Eugenia, podem prejudicar a germinação (Oliveira et al. 2011). No presente trabalho, porém, demonstrou-se que quando o período de tratamento osmótico é mais curto obtêm-se bons resultados, controlando a incidência de fungos e prejudicando muito pouco a germinação de sementes.
Contudo, em geral o tratamento osmótico foi menos eficiente que a termoterapia.

Observou-se que cada tratamento aplicado às sementes teve efeito específico para cada um dos diferentes fungos. Para realizar o controle, tais tratamentos dependem da sensibilidade diferencial ao estresse hídrico entre a semente e o fungo, bem como

Tabela 1. Germinação (\%) e sementes germináveis (\%) de Eugenia brasiliensis submetidas a diferentes tratamentos térmicos (Term-0: sem tratamento térmico; Term-1: $55^{\circ} \mathrm{C} / 30 \mathrm{~min}$; Term-2: $55^{\circ} \mathrm{C} / 150 \mathrm{~min}$ ) e osmóticos (Osm-0: sem tratamento osmótico; Osm-1: -3,4 MPa/7dias; Osm-2: -4,0 MPa/7dias), com ou sem reaplicação após 30 dias de armazenamento e armazenadas por até 60 dias $^{(1)}$. CV: coeficiente de variação.

Table 1. Germination (\%) and germinable seeds (\%) of Eugenia brasiliensis after different heat treatments (Term-0: without heat treatment; Term-1: $55^{\circ} \mathrm{C} / 30 \mathrm{~min}$; Term-2: $55^{\circ} \mathrm{C} / 150 \mathrm{~min}$ ) and osmotic treatments (Osm-0: without osmotic treatment; Osm-1: $-3.4 \mathrm{MPa} / 7$ days; Osm-2: $-4.0 \mathrm{MPa} / 7$ days), with or without reapplication after 30 days, and stored until 60 days ${ }^{(1)}$. $\mathrm{CV}$ : coefficient of variation.

\begin{tabular}{|c|c|c|c|c|c|c|}
\hline \multirow{2}{*}{ Tratamento de sementes } & \multicolumn{3}{|c|}{ Sem Reaplicação } & \multicolumn{3}{|c|}{ Com Reaplicação } \\
\hline & Term-0 & Term-1 & Term-2 & Term-0 & Term-1 & Term-2 \\
\hline & \multicolumn{6}{|c|}{ Sementes germináveis após armazenamento de 30 dias } \\
\hline Osm-0 & $98 \mathrm{aA} a$ & $96 \mathrm{aA} a$ & $98 \mathrm{aA} a$ & $98 \mathrm{aA} a$ & $100 \mathrm{aA} a$ & $100 \mathrm{aA} a$ \\
\hline Osm-1 & $94 \mathrm{aA} a$ & $98 \mathrm{aA} a$ & $98 \mathrm{aA} a$ & $73 \mathrm{bB} b$ & $100 \mathrm{aA} a$ & $90 \mathrm{aA} a$ \\
\hline Osm-2 & $92 \mathrm{aA} a$ & $92 \mathrm{aA} a$ & $96 \mathrm{aA} a$ & $79 \mathrm{bB} b$ & $92 \mathrm{aAB} a$ & $94 \mathrm{aA} a$ \\
\hline \multirow[t]{2}{*}{$\mathrm{CV}$} & $8,26 \%$ & & & & & \\
\hline & \multicolumn{6}{|c|}{ Germinação após armazenamento de 30 dias } \\
\hline Osm-0 & $88 \mathrm{aA} a$ & $90 \mathrm{aA} a$ & $94 \mathrm{aA} a$ & $90 \mathrm{aA} a$ & $94 \mathrm{aA} a$ & $94 \mathrm{aA} a$ \\
\hline Osm-1 & $88 \mathrm{aA} a$ & $94 \mathrm{aA} a$ & $90 \mathrm{aA} a$ & $56 \mathrm{bB} b$ & $77 \mathrm{bA} b$ & $79 \mathrm{abA} a$ \\
\hline Osm-2 & $85 \mathrm{aA} a$ & $85 \mathrm{aA} a$ & $88 \mathrm{aA} a$ & $75 \mathrm{aAB} a$ & $88 \mathrm{bA} a$ & $71 \mathrm{bB} b$ \\
\hline \multirow[t]{3}{*}{$\mathrm{CV}$} & $11,55 \%$ & & & & & \\
\hline & Term-0 & Term-1 & Term-2 & Osm-0 & Osm-1 & Osm-2 \\
\hline & \multicolumn{6}{|c|}{ Sementes germináveis após armazenamento de 60 dias } \\
\hline Sem Reaplicação & $52 \mathrm{~b}$ & $71 \mathrm{~b}$ & $91 \mathrm{a}$ & $85 \mathrm{a}$ & $67 \mathrm{~b}$ & $63 \mathrm{~b}$ \\
\hline Com Reaplicação & $70 \mathrm{a}$ & $94 \mathrm{a}$ & $85 \mathrm{a}$ & $84 \mathrm{a}$ & $81 \mathrm{a}$ & $83 \mathrm{a}$ \\
\hline \multirow[t]{2}{*}{$\mathrm{CV}$} & $11,36 \%$ & & & & & \\
\hline & \multicolumn{6}{|c|}{ Germinação após armazenamento de 60 dias } \\
\hline Sem Reaplicação & $49 \mathrm{a}$ & $65 \mathrm{~b}$ & $87 \mathrm{a}$ & $83 \mathrm{a}$ & $60 \mathrm{a}$ & $58 \mathrm{~b}$ \\
\hline Com Reaplicação & $60 \mathrm{a}$ & $88 \mathrm{a}$ & $72 b$ & $74 \mathrm{a}$ & $70 \mathrm{a}$ & $74 \mathrm{a}$ \\
\hline Osm-0 & $63 \mathrm{aB}$ & $93 \mathrm{aA}$ & $81 \mathrm{aA}$ & & & \\
\hline Osm-1 & $55 \mathrm{aB}$ & $60 \mathrm{bB}$ & $79 \mathrm{aA}$ & & & \\
\hline Osm-2 & $45 \mathrm{aB}$ & $76 \mathrm{abA}$ & $77 \mathrm{aA}$ & & & \\
\hline $\mathrm{CV}$ & $21,85 \%$ & & & & & \\
\hline
\end{tabular}

(1)Médias seguidas pela mesma letra (minúsculas: comparações dentro das colunas; maiúsculas: comparações dentro das linhas; itálicas: comparações entre Com ou Sem Reaplicação para sementes germináveis e germinação aos 30 dias de armazenamento) não diferem entre si pelo Teste de Tukey ao nível de 5\% de probabilidade.

(1) Means sharing the same letter (small letters compare treatments within each column; capital letters compare treatments within each line; italic letters compare treatments of reapplying for 30 days of storage) are not significantly different by Tukey's test at $5 \%$. 
ao binômio tempo-temperatura. A associação dos tratamentos térmicos aos osmóticos possibilitou que os resultados mais favoráveis obtidos, quando aplicados isoladamente, fossem replicados, mostrando-se eficiente para a redução da incidência dos fungos e a manutenção da qualidade fisiológica das sementes. Nas sementes das duas espécies de Eugenia, Botrytis sp., Cladosporium sp. e Pestalotiopsis sp. tiveram sua incidência reduzida e por vezes anulada com a aplicação dos tratamentos associados nas sementes, apresentando incidência próxima e até menor do que nos tratamentos aplicados isoladamente.
Os tratamentos alternativos ao uso do fungicida tiveram um efeito imediato, como é observado, por exemplo, com a redução da incidência de Penicillium sp. em E. brasiliensis. Contudo, seu efeito aparentemente não persiste ao longo do armazenamento das sementes. A reaplicação dos tratamentos mostrou grande potencial para reforçar o efeito de controle sobre os fungos e, talvez, melhores resultados poderão ser obtidos com sucessivas reaplicações.

Os resultados obtidos no presente trabalho permitiram verificar que o tratamento térmico reduz

Tabela 2. Germinação (\%) e sementes germináveis (\%) de Eugenia uniflora submetidas a diferentes tratamentos térmicos (Term-0: sem tratamento térmico; Term-1: $55^{\circ} \mathrm{C} / 120 \mathrm{~min}$; Term-2: $60{ }^{\circ} \mathrm{C} / 120 \mathrm{~min}$ ) e osmóticos (Osm-0: sem tratamento osmótico; Osm-1: -3,4 MPa/7dias; Osm-2: -4,0 MPa/7dias), com ou sem reaplicação após 30 dias de armazenamento e armazenadas por até 60 dias $^{(1)}$. CV: coeficiente de variação.

Table 2. Germination (\%) and germinable seeds (\%) of Eugenia uniflora after different heat treatments (Term-0: without heat treatment; Term-1: $55^{\circ} \mathrm{C} / 120 \mathrm{~min}$; Term-2: $\left.60{ }^{\circ} \mathrm{C} / 120 \mathrm{~min}\right)$ and osmotic treatments (Osm- 0 : without osmotic treatment; Osm-1: $-3.4 \mathrm{MPa} / 7$ days; Osm-2: $-4.0 \mathrm{MPa} / 7$ days), with or without reapplication after 30 days, and stored until 60 days ${ }^{(1)}$. $\mathrm{CV}$ : coefficient of variation.

\begin{tabular}{|c|c|c|c|c|c|c|c|c|c|c|c|c|}
\hline \multirow{2}{*}{$\begin{array}{l}\text { Tratamento de } \\
\text { sementes }\end{array}$} & \multicolumn{5}{|c|}{ Sementes germináveis } & \multicolumn{7}{|c|}{ Germinação } \\
\hline & \multicolumn{2}{|c|}{ Term- 0} & \multicolumn{2}{|l|}{ Term-1 } & \multicolumn{2}{|l|}{ Term-2 } & \multicolumn{2}{|c|}{ Term-0 } & \multicolumn{2}{|c|}{ Term-1 } & \multicolumn{2}{|c|}{ Term-2 } \\
\hline & \multicolumn{12}{|c|}{ Inicial } \\
\hline Osm-0 & 85 & & 83 & & 25 & & 81 & & 71 & & 12 & \\
\hline Osm-1 & 90 & & 88 & & 23 & & 79 & & 77 & & 8 & \\
\hline Osm-2 & 81 & & 81 & & 23 & & 71 & & 67 & & 8 & \\
\hline Médias & 85 & $\mathrm{~A}$ & 84 & $\mathrm{~A}$ & 24 & $\mathrm{~B}$ & 77 & A & 72 & A & 10 & B \\
\hline \multirow[t]{2}{*}{$\mathrm{CV}$} & $16,44 \%$ & & & & & & $20,94 \%$ & & & & & \\
\hline & \multicolumn{12}{|c|}{ Armazenamento de 30 dias } \\
\hline Osm-0 & 81 & $\mathrm{aA}$ & 89 & $\mathrm{aA}$ & 0 & bB & 79 & $\mathrm{aA}$ & 89 & $\mathrm{aA}$ & 0 & $\mathrm{bB}$ \\
\hline Osm-1 & 84 & $\mathrm{aA}$ & 88 & $\mathrm{aA}$ & 19 & $\mathrm{aB}$ & 83 & $\mathrm{aA}$ & 80 & $\mathrm{aA}$ & 14 & $\mathrm{aB}$ \\
\hline Osm-2 & 89 & $\mathrm{aA}$ & 88 & $\mathrm{aA}$ & 28 & $\mathrm{aB}$ & 83 & $\mathrm{aA}$ & 81 & $\mathrm{aA}$ & 17 & $\mathrm{aB}$ \\
\hline \multirow[t]{2}{*}{$\mathrm{CV}$} & $16,12 \%$ & & & & & & $18,64 \%$ & & & & & \\
\hline & \multicolumn{12}{|c|}{ Armazenamento de 60 dias } \\
\hline Osm-0 & 71 & & 90 & & 0 & & 85 & & 85 & & 2 & \\
\hline Osm-1 & 85 & & 81 & & 4 & & 81 & & 81 & & 2 & \\
\hline Osm-2 & 75 & & 83 & & 4 & & 81 & & 77 & & 0 & \\
\hline Médias & 80 & A & 83 & A & 2 & $\mathrm{~B}$ & 74 & A & 78 & $\mathrm{~A}$ & 1 & B \\
\hline $\mathrm{CV}$ & $16,66 \%$ & & & & & & $20,04 \%$ & & & & & \\
\hline
\end{tabular}

Médias seguidas pela mesma letra (minúsculas: comparações dentro das colunas; maiúsculas: comparações dentro das linhas) não diferem entre si pelo Teste de Tukey ao nível de 5\% de probabilidade. Não houve efeito dos tratamentos osmóticos sobre os valores iniciais e após 60 dias de armazenamento.

Means sharing the same letter (small letters compare treatments within each column; capital letters compare treatments within each line) are not significantly different by Tukey's test at $5 \%$. There was no effect of osmotic treatments on initial values as well as after 60 days of storage. 
a incidência da maioria dos fungos presentes em sementes de Eugenia, mas pode potencializar a incidência de Penicillium sp. e apresenta baixo ou nenhum controle sobre Fusarium sp. Já o tratamento osmótico, empregando curtos períodos de exposição das sementes à solução osmótica, foi eficiente em reduzir a incidência de alguns fungos, principalmente Penicillium sp., mas no geral é menos eficiente que o térmico. A associação dos tratamentos apresentou resultados satisfatórios, contudo mais estudos devem ser realizados incluindo diferentes combinações de temperatura, tempo de exposição e sequência de aplicação. A reaplicação dos tratamentos mostrou-se potencialmente eficiente, compensando a falta de efeito residual obtida com fungicidas.

\section{Agradecimentos}

Os autores agradecem ao Dr. João J. D. Parisi e a Dra. Rosely A. Piccolo Grandi, pela assistência na identificação dos fungos; ao Conselho Nacional de Desenvolvimento Científico e Tecnológico - CNPq, pelo auxílio financeiro ao projeto e pela bolsa de produtividade em pesquisa ao segundo autor; à Coordenação de Aperfeiçoamento de Pessoal de Nível Superior - CAPES, pela bolsa de Mestrado fornecida à primeira Autora; ao Programa de Pós-Graduação em Biodiversidade Vegetal e Meio Ambiente do Instituto de Botânica, pela oportunidade de realização do Curso de Mestrado; ao Instituto de Botânica, pela autorização para a coleta dos frutos.

\section{Literatura citada}

Amador, T.S. \& Barbedo, C.J. 2011. Potencial de inibição da regeneração de raízes e plântulas em sementes germinantes de Eugenia pyriformis. Pesquisa Agropecuária Brasileira 46: 814-821.

Andréo, Y., Nakagawa, J. \& Barbedo, C.J. 2006. Mobilização de água e conservação da viabilidade de embriões de sementes recalcitrantes de ingá (Inga vera Willd. subsp. affinis (DC.) T. D. Pennington). Revista Brasileira de Botânica 29: 309-318.

Anguelova-Merhar, V.S., Calistru, C. \& Berjak, P. 2003. A study of some biochemical and histopathological responses of wet-store recalcitrant seeds of Avicennia marina infected by Fusarium moniliforme. Annals of Botany 92: 401-408.

Barbedo, C.J., Centeno, D.C. \& Figueiredo-Ribeiro, R.C.L. 2013. Do recalcitrant seeds really exist? Hoehnea 40: 583-593.

Barnett, H.L. \& Hunter, B.B. 1999. Ilustrated genera of imperfect fungi. 3ed. Burgess Publishing Company, Minneapolis.
Beckman, C.H. \& Roberts, E.M. 1995. On the nature and genetics basis for resistance and tolerance to fungal wilt diseases of plants. Advances in Botanical Research 21: 35-77.

Berjak, P. 1996. The role of micro-organisms in deterioration during storage of recalcitrant and intermediate seeds. In: A.S. Quedraogo, N.K. Poulsen \& F. Stubsgaard (eds.). Workshop on Improved Methods for Handling and Storage of Intermediate/Recalcitrant Tropical Forest Tree Seeds. IPGRI, Rome, pp.121-126.

Bettiol, W. \& Ghini, R. 2003. Proteção de plantas em sistemas agrícolas alternativos. In: C. Campanhola \& W. Bettiol (eds.). Métodos alternativos de controle fitossanitário. Embrapa Meio Ambiente, Jaguariúna, pp. 79-96.

Brasil. 2009. Regras para análise de sementes. Ministério da Agricultura, Pecuária e Abastecimento, Secretaria de Defesa Agropecuária, Coordenação Geral de Apoio Laboratorial, Brasília.

Calistru, C., Mclean, M., Pammenter, N.M. \& Berjak, P. 2000. The effects of mycofloral infection on the viability and ultrastructure of wet-stored recalcitrant seeds of Avicennia marina (Forssk) Vierh. Seed Science Research 10: 341-352.

Civello, P.M., Martínez, G.A., Chaves, A.R. \& Añon, M.C. 1997. Heat treatments delay ripening and postharvest decay of strawberry fruit. Journal of Agricultural and Food Chemistry 45: 4589-4594.

Delgado, L.F. \& Barbedo, C.J. 2007. Tolerância à dessecação de sementes de espécies de Eugenia. Pesquisa Agropecuária Brasileira 42: 265-272.

Delgado, L.F. \& Barbedo, C.J. 2012. Water potential and viability of seeds of Eugenia (Myrtaceae), a tropical tree species, based upon different levels of drying. Brazilian Archives of Biology and Technology 55: 583-590.

Delgado, L.F., Mello, J.I. \& Barbedo, C.J. 2010. Potential for regeneration and propagation from cut seeds of Eugenia (Myrtaceae) tropical tree species. Seed Science and Technology 38: 624-634.

Dhingra, O.D. 2005. Teoria da transmissão de patógenos fúngicos por sementes. In: L. Zambolim (ed.). Sementes: qualidade fitossanitária. UFV, DFP, Viçosa, pp. 75-112.

Kohama, S., Maluf, A.M., Bilia, D.A.C. \& Barbedo, C.J. 2006. Secagem e armazenamento de sementes de Eugenia brasiliensis Lam. (grumixameira). Revista Brasileira de Sementes 28: 72-78.

Mendes, M.A.S., Lima, P.M.M.P., Fonseca, J.N.L. \& Santos, M.F. 2001. Erradicação de Fusarium oxysporum em sementes de alfafa utilizando termo e quimioterapia. Fitopatologia Brasileira 26: 148-152.

Michel, B.E. \& Kaufmann, M.R. 1973. The osmotic potential of polyethylene glicol 6000. Plant Physiology 51: 914-916.

Mycock, D.J. \& Berjak, P. 1990. Fungal contaminants associated with several homoiohydrous (recalcitrant) seed species. Phytophylactica 22: 413-418. 
Mycock, D.J. \& Berjak, P. 1995. The implications of seed associated mycoflora during storage. In: J. Kigel \& G. Galili (eds.). Seed development and germination. Marcel Dekker Inc., New York, pp.747-766.

Oliveira, C.F., Oliveira, D.C., Parisi, J.J.D. \& Barbedo, C.J. 2011. Deterioração de sementes de espécies brasileiras de Eugenia em função da incidência e do controle de fungos. Revista Brasileira de Sementes 33: 520-532.

Parisi, J.J.D., Santos, A.F. \& Menten, J.O.M. 2011. Tratamento de sementes florestais. In: A.F. dos Santos, J.J.D. Parisi \& J.O.M. Menten (eds). Patologia de sementes florestais. Embrapa Florestas, Colombo, pp. 105-114.

Parisi, J.J.D., Biagi, J.D., Barbedo, C.J. \& Medina, P.F. 2013. Viability of Inga vera Willd. subsp. affinis (DC.) T. D. Penn. embryos according to the maturation stage, fungal incidence, chemical treatment and storage. Journal of Seed Science 35: 70-76.

Santana, D.G. \& Ranal, M. 2004. Análise da germinação: um enfoque estatístico. Editora Universidade de Brasília, Brasília, pp. 248.
Schmeda-Hirschmann, G., Theoduloz, C., Franco, L., Ferro, E.B. \& Arias, A.R. 1987. Preliminary pharmacological studies on Eugenia uniflora leaves: xanthine oxidase inhibitory activity. Journal of Ethnopharmacology 21: 183-186.

Silva, A.M.S., Carmo, M.G.F., Olivares, F.L. \& Pereira, A.J. 2002. Termoterapia via calor seco no tratamento de sementes de tomate: eficiência na erradicação de Xanthomonas campestris pv. vesicatoria e efeitos sobre a semente. Fitopatologia Brasileira 27: 586-593.

Silva, P.P., Freitas , R.A. \& Nascimento, W.M. 2011. Detection of tomato mosaic virus in tomato seed and treatment by thermotherapy. Acta Horticulturae 917: 303-308.

Splittstoesser, D.F. \& Churey, J.J. 1991. Reduction of heat resistance of Neosartorya fischeri ascospores by sulfur dioxide. Journal of Food Science 556: 876-877.

Sutherland, J.R., Dikmann, M. \& Berjak, P. 2002. Forests tree seed health for germplasm conservation. IPGRI, Rome.

Tanaka, M.A.S., Ito, M.F., Braga, C.A.S. \& Armond, G. 2003. Tratamento térmico solar da água para controle de fitopatógenos. Fitopatologia Brasileira 28: 386-393. 